\title{
Recent progress on the study of nucleon structure from lattice QCD and future perspectives
}

\author{
Constantia Alexandrou ${ }^{1,2 \star}$ \\ 1 Department of Physics, University of Cyprus, P.O. Box 20537, 1678 Nicosia, Cyprus \\ 2 Computation-based Science and Technology Research Center, The Cyprus Institute, 20 \\ Kavafi Str., Nicosia 2121, Cyprus \\ * alexand@ucy.ac.cy
}

November 23, 2019

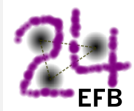

Proceedings for the 24th edition of European Few Body Conference, Surrey, UK, 2-4 September 2019

\begin{abstract}
We review the progress achieved within the last five years in the simulations of lattice QCD, as well as in the analysis for selected quantities probing nucleon structure. In particular, we discuss results on the nucleon electromagnetic form factors, $\sigma$-terms, the momentum fraction carried b quark in the nucleon and the helicity and transversity. All quantities are obtained using simulations generated with quark masses fixed to their physical values. In addition, we review the on-going effort to extract parton distribution functions (PDFs) directly from lattice QCD using the quasi-PDFs approach.
\end{abstract}

\section{Introduction}

Understanding the structure of the nucleon in terms of its fundamental constituents, the quarks and gluons, is an overarching objective of current and future experiments at major facilities world-wide, such as Jefferson lab, PSI, RHIC, LHC, DESY, and the planned Electron Ion Collider (EIC). A rich experimental program is underway to unravel the valence quark distribution functions at Jefferson lab with the $12-\mathrm{GeV}$ upgrade. The signature of sea quarks is being studied at Fermilab with a polarized beam and target as well as at RHIC shedding light on the orbital angular momentum and spin of light sea quarks. Generalized Parton Distributions (GPDs) investigated through the analysis of hard exclusive processes at HERA, COMPASS and CLAS12, allow one to construct the three-dimensional spatial map. This program will be greatly reinforced by the future experiments at Jefferson Lab for valence quarks, while for the gluons and sea quarks at the Electron-Ion Collider providing a complete three-dimensional mapping of the nucleon [1].

Lattice QCD, has seen remarkable progress in recent years producing simulations at the physical values of the quark masses. This allows to obtain nucleon observables and properties without the need for a chiral extrapolation, thus eliminating a major source of an uncontrolled systematic error. In addition, theoretical progress has enabled exploratory studies of the parton distribution functions themselves directly from lattice QCD [2]. This is to be compared to the traditional approach of calculating their lower moments. In this presentation, we review results on a number of nucleon quantities that related to the aforementioned experiments. 


\section{The lattice QCD formalism}

Gauge invariant quantities are computed by evaluating the path integral

$$
\langle\mathcal{O}\rangle=\frac{1}{Z} \int \mathcal{D}[U] \mathcal{O}\left(D^{-1}[U], U\right) \prod_{f=u, d, s, c} \operatorname{Det}(D[U]) e^{-S[U]},
$$

over gauge configurations $U$, after integrating over the fermionic degrees of freedom. A Wick rotation into imaginary time is already performed. $S[U]$ is the exact gauge action of QCD and $D[U]$ is the exact fermionic determinant that occurs after integrating over the fermions and generates sea quark loops. $D^{-1}$ is the quark propagator arising from the fermions in the operator. In order to evaluate the path integral of Eq. (1), one defines the theory on a discrete 4-dimensional Euclidean lattice and considers a finite volume $V$. The functional integral over the gauge degrees of freedom is then amenable to a numerical simulation. The input required is the same as for QCD, namely the quark masses and the coupling constant. The latter is directly related to the lattice spacing $a$ through the $\beta$-function. Therefore, one needs four physical quantities to fix the light, strange and charm quark masses and an additional one for the lattice spacing.

There are several ways to put the fermions on a discrete lattice that leads to different ways of representing $D$, each one having its own advantages and disadvantages. The main ones are: i) Wilson-type fermions that include the clover and twisted mass formulations, ii) staggered fermions, iii) domain wall fermions, and iv) overlap fermions, with associated collaborations. In the continuum limit these different formulations should all agreed.

A lattice QCD computation is comprised of three main steps [3]:

- Generation of the gauge configurations $\{U\}$. This proceeds via a Monte-Carlo sampling that includes all the effects of gluonic self interactions and the interactions of gluons and sea quarks. The ensembles are parameterized by the value of the strong coupling or lattice spacing and the masses of the sea quarks. Within each ensemble the configurations are generated via a Markov process, sequentially. This first step requires access to leadership computers and larger resources. These gauge ensembles can then be used to evaluate any quantity $\mathcal{O}$. Several ensembles with different lattice spacings and volumes at fix quark masses are required in order to take the continuum and infinite volume limits.

- The second step is the computation of $D^{-1}$, which is the quark propagator. The quark propagators are contracted into correlation functions according to the operator $\mathcal{O}$. Since the size of the fermionic metric $D$ is large, this step also requires leadership computers and large resources to perform the large number of inversions, which however can be done independently on each gauge configuration.

- The third step involves the analysis of the resulting correlation functions to extract the expectation value of $\mathcal{O}$. This analysis can be done on smaller systems.

The work-flow of a typical lattice QCD computation for baryon structure is shown schematically in Fig. 1. The three-point correlation functions are divided into connected when a probe couples to a valence quark and to disconnected when it couples to a sea quark or a gluon.

As already mentioned, lattice QCD simulations are being performed with quark masses fixed to their physical values. This was achieved by developing multi-grid algorithms that make inversions at physical light quark mass faster. As can be seen in Fig. 2, the cost for inversions remain constant with decreasing the value of the quark mass, making simulations and their analysis possible. 


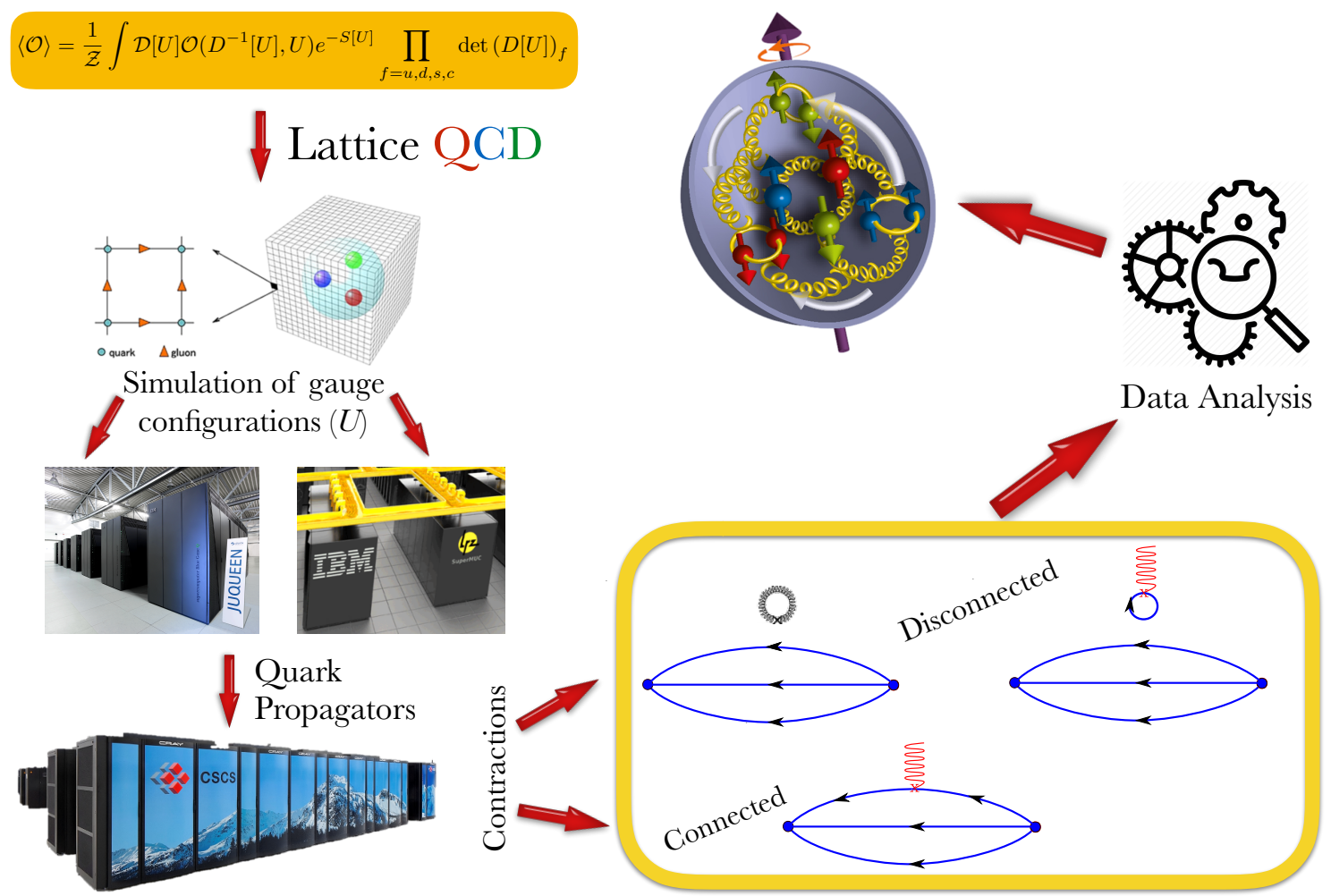

Figure 1: A typical work-flow for a lattice QCD hadron structure computation. The diagrams shown in the lower left box correspond to the connected and disconnected three-point correlators needed for baryon structure studies.
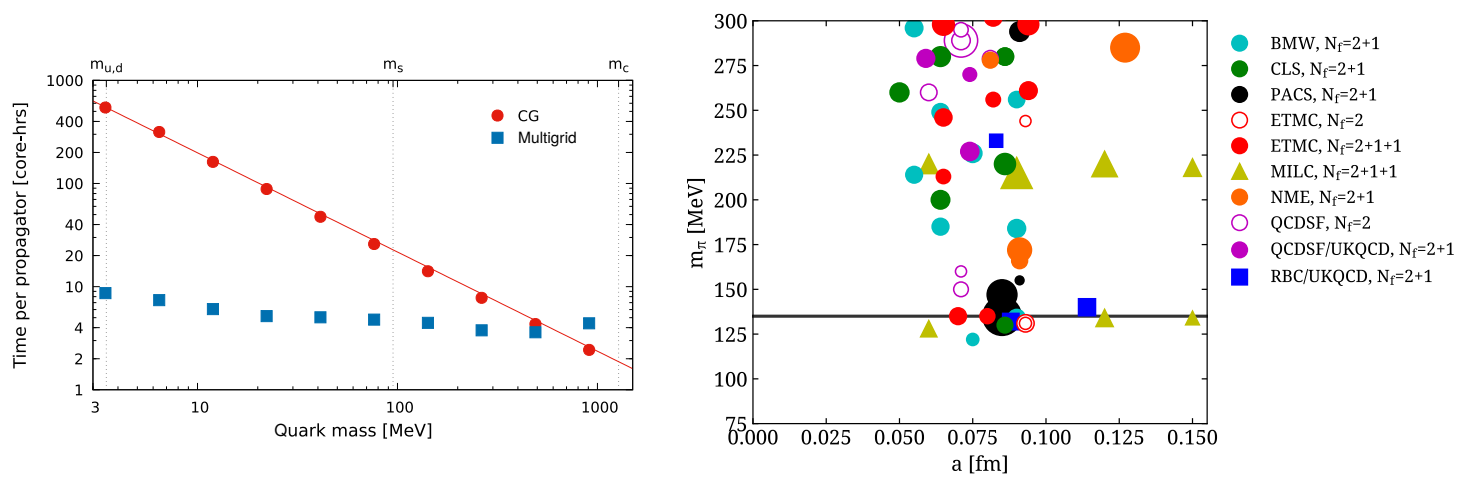

Figure 2: Left: Time to solution for the computation of $D^{-1}$ using the conjugate gradient (red points) and the 3-level Adaptive Aggregation-based Domain Decomposition Multigrid (DD- $\alpha \mathrm{AMG}$ ) for twisted mass fermion [4,5]. Right: A summary of zero-temperature simulations by various collaborations that include Clover, Twisted Mass, Staggered and Domain Wall fermions.

Although lattice QCD provides an exact formalism for solving QCD, one needs a careful study of systematic errors. In the past, not having simulations at physical values of the light quark mass, necessitated a chiral extrapolation. For the pion sector such an extrapolation using NLO SU(2) chiral perturbation theory works well for pion masses $m_{\pi}<250 \mathrm{MeV}$ [6]. In the nucleon sector chiral extrapolation is more problematic and previously introduced an 
uncontrolled systematic error [7]. With simulations with physical pion mass this systematic error has been eliminated. Therefore, in what follows we will focus on results obtained using near physical pion mass, since we will focus on baryons. Other systematic effects that need to be investigated are:

- Discretisation effects: Since the computation is done at finite lattice spacing $a$ one needs to take the continuum limit. This requires simulations at least three values of $a$ at fixed quark masses and volume.

- Finite volume effects: A numerical evaluation is necessarily done using a finite volume. At least three volumes would be required at fixed quark masses and $a$ to take the infinite volume limit.

- Renormalization: Matrix elements computed on the lattice must be properly renormalized in order to compare with what is measured in the laboratory. In state-of-the-art computations renormalization is carried out non-perturbatively. However, for gluonic quantities and where there is mixing non-perturbative renormalization is still difficult and it is an on-going process.

- Ground state identification: Extracting the ground state from a tower of higher excited states needs large Euclidean time resulting in large gauge noise and difficult identification of ground state properties. Furthermore, for a class of quantities, such as for the direct computation of PDFs, one needs to boost the hadron to large momentum. This introduces large statistical errors requiring very large statistics and development of noise reduction techniques.

\section{Low-lying baryon spectrum}

The simplest quantities to calculate in lattice QCD are hadrons masses. For this class of observables the aforementioned systematics have been taken into account. Hadron masses are
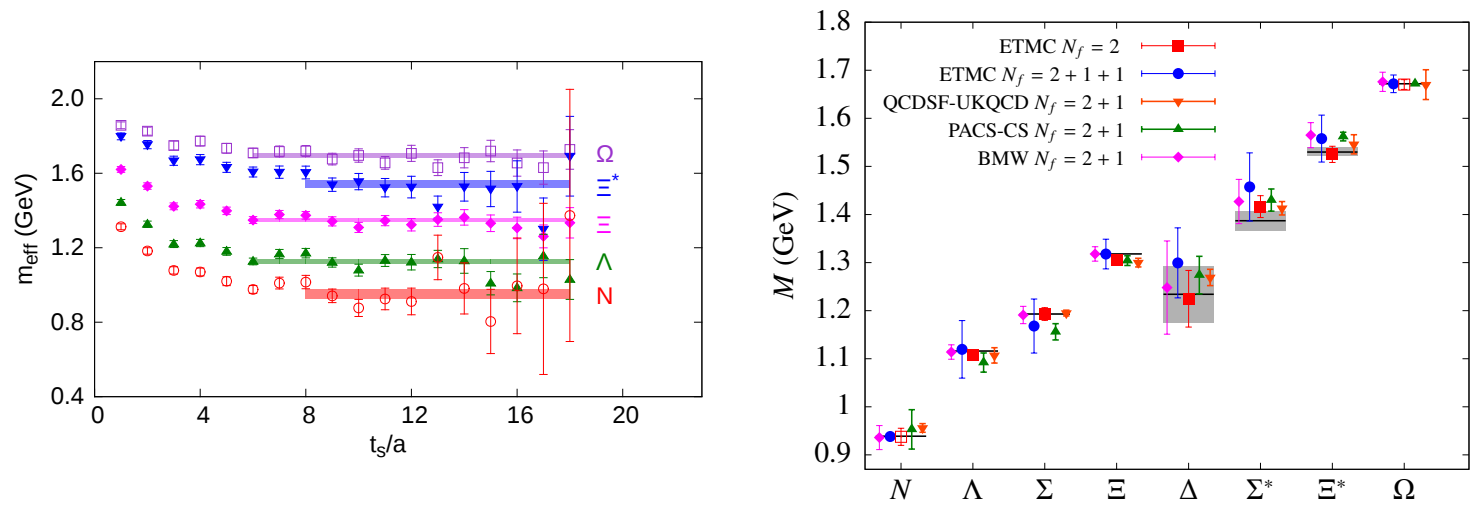

Figure 3: Left: The effective mass of low-lying baryons. Right: the mass of the low-lying octet and decuplet baryons from various collaborations extrapolated to the continuum limit. The horizontal bands are the experimental values. Open symbols indicate the input used to fix the lattice spacing (nucleon) and strange quark mass $\left(\Omega^{-}\right)$.

extracted from two-point correlation functions in the large Euclidean time $t_{s}$

$$
C_{2 \mathrm{pt}}\left(\vec{q}, t_{s}\right)=\sum_{\vec{x}_{s}} e^{-i \vec{x}_{s} \cdot \vec{q}}\left\langle J\left(\vec{x}_{s}, t_{s}\right) \bar{J}(0)\right\rangle=\sum_{n=0, \cdots, \infty} A_{n} e^{-E_{n}(\vec{q}) t_{s}} \stackrel{t_{s} \rightarrow \infty}{\longrightarrow} A_{0} e^{-E_{0}(\vec{q}) t_{s}}
$$


taking $\vec{q}=\overrightarrow{0}$. The effective mass defined as the ratio $m_{\text {eff }}\left(\overrightarrow{0}, t_{s}\right)=\ln \left[G\left(\overrightarrow{0}, t_{s}\right) / G\left(\overrightarrow{0}, t_{s}+a\right)\right]$, yields the mass of the lowest lying hadron of the quantum numbers of $J$. We show in Fig. 3 , the effective mass of the low lying baryons extracted by various collaborations. The lattice QCD results agreed with the experimental values providing a validation of the lattice QCD approach. In Fig. 4, we show the masses of the spin- $1 / 2$ and $3 / 2$ charmed baryons. The doubly charmed $\Xi_{c c}$ was predicted by lattice QCD to have larger mass than claimed by SELEX. LHCb at CERN confirmed the lattice QCD prediction illustrating the predictive power of lattice QCD.
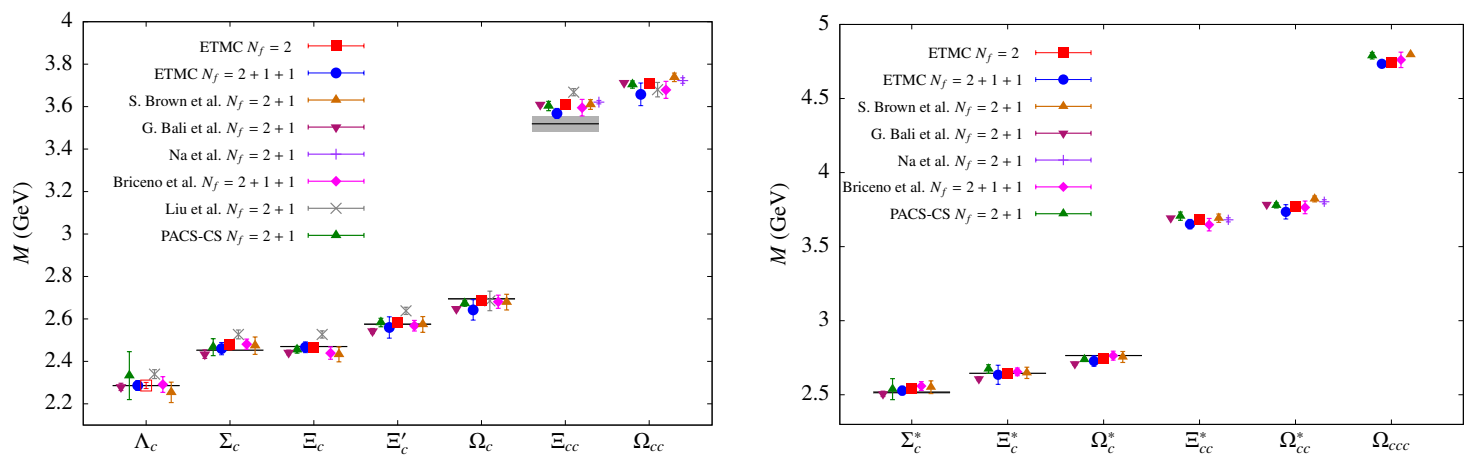

Figure 4: Left: Masses of spin $1 / 2$ baryons. The mass of $\Xi_{c c}$ was correctly predicted by lattice QCD and confirmed by LHCb [8] and higher than the value by SELEX [9], shown by the grey band. Right: Masses of spin-3/2 baryons [10]. The mass of $\Lambda_{c}$ is used to fix the charm quark mass.

\section{Nucleon structure}

In order to calculate matrix elements one compute the following three-point function

$$
C_{3 \mathrm{pt}}^{\mu v}\left(\Gamma ; \vec{q}=0, t_{s}, t_{\mathrm{ins}}\right)=\sum_{\vec{x}_{\mathrm{ins}}, \vec{x}_{s}} \operatorname{Tr}\left[\left\langle\Gamma J_{N}\left(t_{s}, \vec{x}_{s}\right) \mathcal{O}^{\mu v}\left(t_{\mathrm{ins}}, \vec{x}_{\mathrm{ins}}\right) \bar{J}_{N}\left(t_{0}, \vec{x}_{0}\right)\right\rangle\right],
$$

where you consider an operator $\mathcal{O}^{\mu v}$ probing the nucleon. The three-point function has connected and disconnected diagrams shown diagrammatically in Fig. 5.
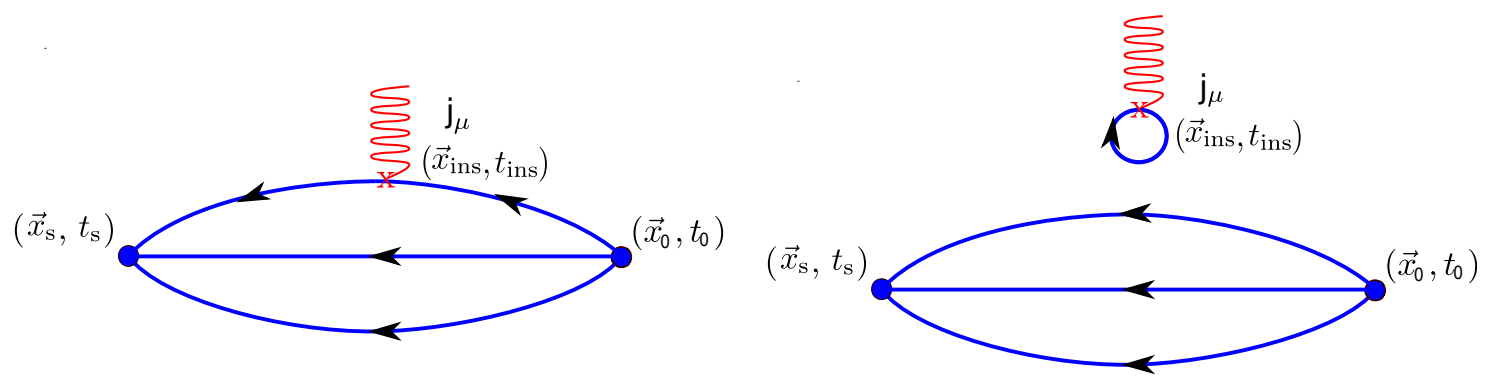

Figure 5: A diagrammatic representation of the connected (left) and disconnected (right) three-point functions. A state with the quantum numbers of the nucleon is created at time zero (source) that propagates in Euclidean time and annihilated at time $t_{s}$ (sink). The probe couples to a quark at time $t_{\text {ins }}$.

As in the case of the effective mass and considering for simplicity $\vec{q}=0$, we construct a ratio

$$
R^{\mu v}\left(\Gamma ; \vec{q}=\overrightarrow{0}, t_{s}, t_{\text {ins }}\right)=\frac{C_{3 \mathrm{pt}}^{\mu v}\left(\Gamma, \vec{q}=0, t_{s}, t_{\text {ins }}\right)}{C_{2 \mathrm{pt}}\left(\Gamma_{0}, t_{s}\right)} \longrightarrow \mathcal{M}+\mathcal{O}\left(e^{-\Delta E\left(t_{s}-t_{\mathrm{ins}}\right)}\right)+\mathcal{O}\left(e^{-\Delta E t_{\text {ins }}}\right),
$$


which in the large Euclidean time becomes time independent (plateau), yielding the nucleon matrix element $\mathcal{M}$. However, the statistical errors grow exponentially with the time separation and making the detection of excited states difficult. We thus include in the fit the first excited state (two-state fit) or even a second state if the lattice QCD data are accurate enough. Another approach is to sum the ratio to obtain

$$
\sum_{t_{\text {ins }}=a}^{t_{s}-a} R^{\mu v}\left(\Gamma ; \vec{q}=\overrightarrow{0}, t_{s}, t_{\text {ins }}\right) \longrightarrow c+\mathcal{M} t_{s}+\mathcal{O}\left(e^{-\Delta E t_{s}}\right) .
$$

By fitting the summed ratio linearly with $t_{s}$ one can extract $\mathcal{M}$. This approach is referred to as the summation method. We typically use a combination of these three approaches to check for the suppression of excited states.

\subsection{Moments of parton distribution functions}

Generalized parton distribution functions are light-cone correlation functions written as

$$
F_{\Gamma}\left(x, \xi, q^{2}\right)=\frac{1}{2} \int \frac{d \lambda}{2 \pi} e^{i x \lambda}\left\langle p^{\prime}\left|\bar{\psi}(-\lambda k / 2) \mathcal{G} e^{i g \int_{-\lambda / 2}^{\lambda / 2} d \alpha k \cdot A(k \alpha)} \psi(\lambda k / 2)\right| p\right\rangle,
$$

where $q=p^{\prime}-p$ is the momentum transfer, $\bar{P}=\left(p^{\prime}+p\right) / 2, k$ is a light-cone vector and $\bar{P} \cdot k=1$. Expansion of the light cone operator leads to a tower of local operators $\mathcal{O}^{\mu \mu_{1} \ldots \mu_{n}}$ and thus to the computation of nucleon matrix elements of quark bilinears $\left\langle N\left(p^{\prime}, s^{\prime}\right)\left|\mathcal{O}_{\mathcal{G}}^{\mu_{1} \cdots \mu_{n}}\right| N(p, s)\right\rangle$. Depending on the $\mathcal{G}$-structure we have three classes of Mellin moments:

i) Unpolarized: $\left.\mathcal{O}_{V}^{\mu \mu_{1} \cdots \mu_{n}}=\bar{\psi}(x) \gamma^{\left\{\mu_{i} \stackrel{\leftrightarrow}{D}\right.} \mu^{\mu_{1}} \ldots i \stackrel{\leftrightarrow}{D}{ }^{\mu_{n}}\right\} \psi(x)$, the lowest two of which are $g_{V}^{q}$ for $n=0$ and $\langle x\rangle_{q}$ for $n=1$

ii) Helicity: $\left.\mathcal{O}_{A}^{\mu \mu_{1} \cdots \mu_{n}}=\bar{\psi}(x) \gamma^{\left\{\mu_{i} \stackrel{\leftrightarrow}{D}\right.}{ }^{\mu_{1}} \ldots i \stackrel{\leftrightarrow}{D} \mu_{n}\right\} \gamma_{5} \psi(x)$, the lowest two of which are $g_{A}^{q}$ for $n=0$ and $\langle x\rangle_{\Delta q}$ and $n=1$

iii) Transversity: $\left.\mathcal{O}_{T}^{v \mu \mu_{1} \cdots \mu_{n}}=\bar{\psi}(x) \sigma^{\left\{v, \mu_{i} \stackrel{\leftrightarrow}{D} \mu_{1}\right.} \ldots i \stackrel{\leftrightarrow}{D} \mu_{n}\right\} \frac{\tau^{a}}{2} \psi(x)$, the lowest two of which are $g_{T}^{q}$ for $n=0$ and $\langle x\rangle_{\Delta q}$ for $n=1$.

The first two Mellin moments can readily be computed within lattice QCD and there is a long history of such computations. However, it is only recently that we have results directly at the physical point i.e. using simulations with $m_{\pi} \sim 135 \pm 10 \mathrm{MeV}$.

In order to benchmark our lattice QCD formalism and analysis, we examine the case of the isovector axial charge of the nucleon $g_{A}^{u-d}$, which is very accurately measured from neutron $\beta$ decay. This is the first Mellin moment of the helicity PDF. For the unpolarized, since the electromagnetic current is conversed $g_{V}^{u-d}=1$ by symmetry. In contrast, the transversity isovector charge $g_{T}^{u-d}$ is poorly known and it is an example where lattice QCD can provide valuable input. In Fig. 6, we show the analysis for determining the nucleon matrix element that yields $g_{A}^{u-d}$. As can be seen, the ground state matrix element $\mathcal{M}$, determined by the asymptotic plateau value (one-state fit), the two- and three- state fits and the summation method, agrees with the experimental value. This is an example of an analysis carried out using one ensemble of twisted mass fermions with the light, strange and charm quark masses fixed to their physical values [11]. This so-called cB211.072.64 ensemble has $a=0.08 \mathrm{fm}$, $m_{\pi}=139 \mathrm{MeV}$, spatial length $L / a=64$ and $L m_{\pi}=3.6$. The action is $\mathcal{O}(a)$-improved so finite lattice spacing effects are expected to be small. This was indeed shown by previous studies using twisted mass ensembles simulated with heavier than physical pion mass. 


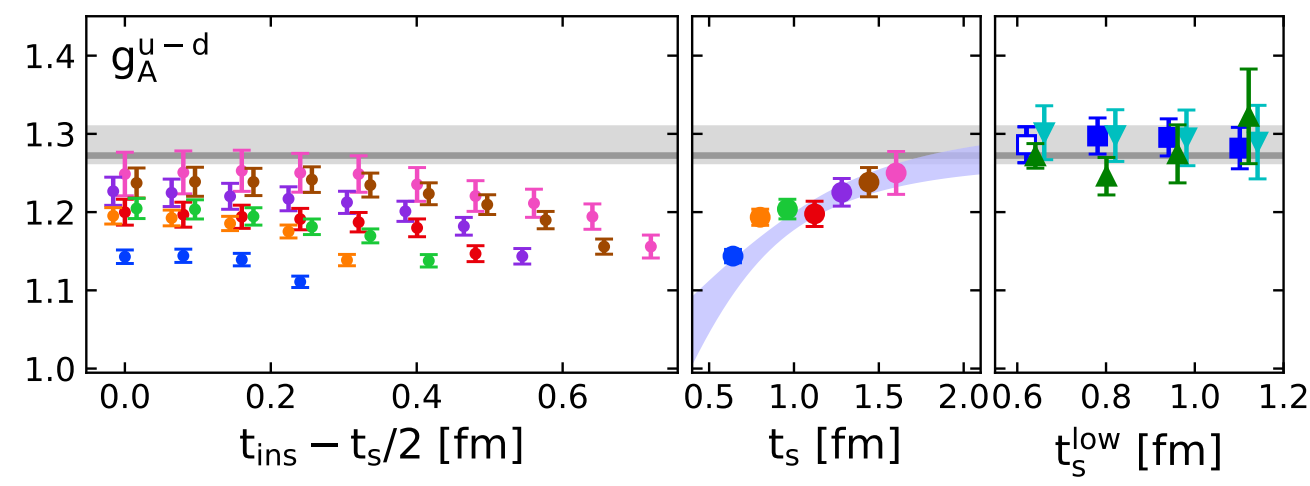

Figure 6: Left: The ratio of Eq. (3) as a function of the time separation $t_{s}$ and the time of the inversion $t_{\text {ins }}$. Center: the values extracted from fitting the data of the left panel to a constant assuming ground state dominance. The blue band is the result of a two-state fit. Right: The values of $\mathcal{M}$ extracted from a two-state fit as a function of the lower value of $t_{s}$ used in the fit, $t_{s}^{\text {low }}$ (filled blue squares) and from three-state fits (light blue down triangles). The filled green triangles are the values of $\mathcal{M}$ extracted using the summation method of Eq. (4). The open blue square is the selected value for $\mathcal{M}$ with the associated error shown with the grey band across the three panels. The darker band shows the PDG value of $g_{A}^{u-d}=1.273(2)$.
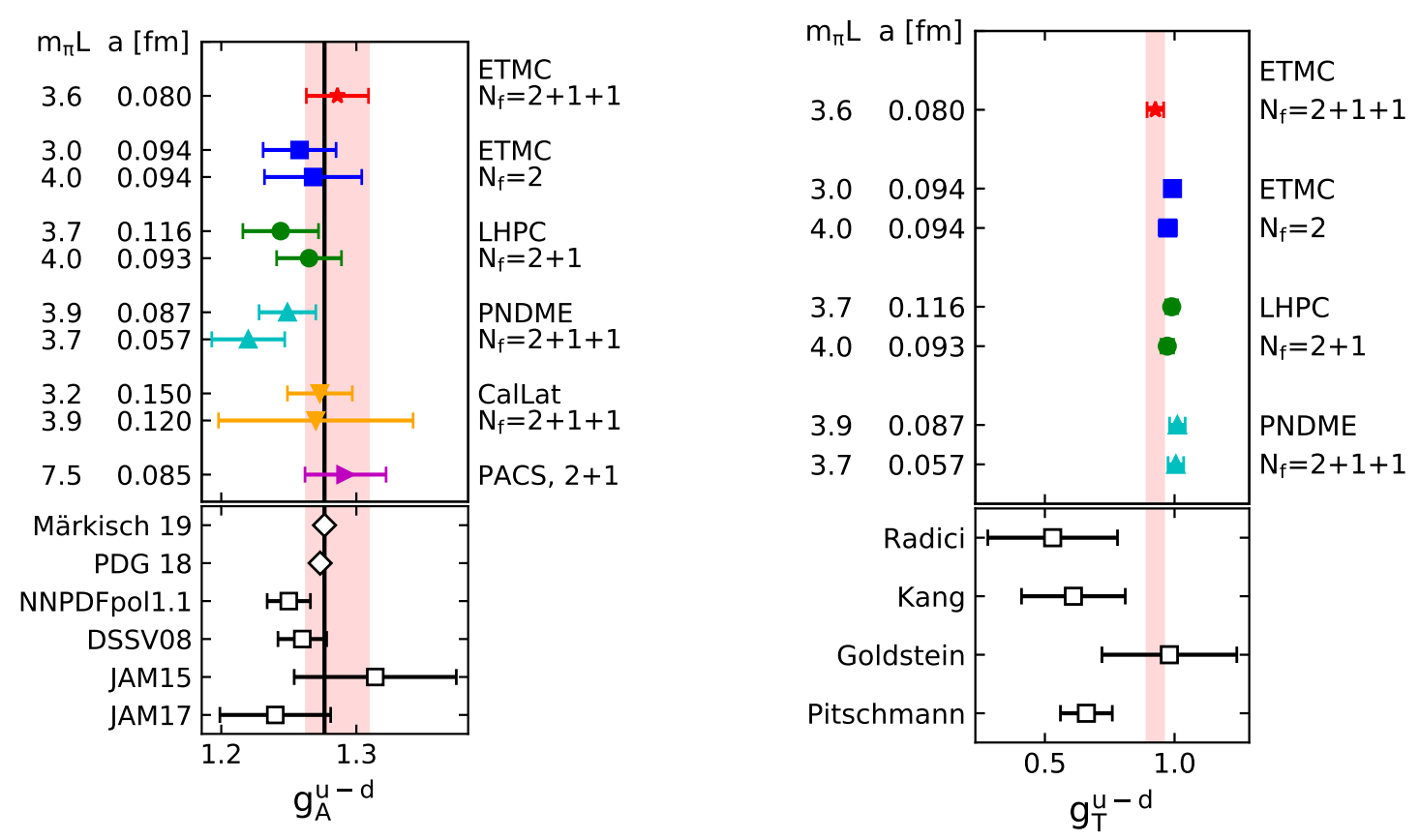

Figure 7: A comparison of recent lattice QCD and phenomenological results on $g_{A}^{u-d}$ (left) and $g_{T}^{u-d}$ (right). The error band is the one associate with the analysis of the twisted mass ensemble cB211.072.64. Open symbols are from phenomenological analysis of experimental data.

A similar analysis is done to extract the isovector tensor charge $g_{T}^{u-d}$. We find a value of $g_{T}^{u-d}=0.926(32)$ in the $\overline{\mathrm{MS}}$ scheme at $\mu=2 \mathrm{GeV}$, providing a more precise value as compared to $0.53 \pm 0.25$ extracted by analyzing experimental data [12].

In Fig. 7 we show results for $g_{A}^{u-d}$ and $g_{T}^{u d-}$ using the cB211.072.64 ensemble in comparison with two additional ensembles of twisted mass fermions with dynamical light quarks, 
lattice spacing $a=0.094 \mathrm{fm}$ and $L m_{\pi}=3$ and $L m_{\pi}=4$, as well as from other collaborations that analyzed ensembles simulated with approximately physical values of the pion mass. As can be seen, there is very good agreement among all lattice computations, which points to small finite $a$ and volume effects, since the continuum and infinite volume limits are not performed.

GPDs can be decomposed Lorentz invariant functions known as generalized form factors (GFFs). For the unpolarized PDF the decomposition is

$\left\langle N\left(p^{\prime}, s^{\prime}\right)\left|\mathcal{O}_{\mathrm{V}}^{\mu \nu}\right| N(p, s)\right\rangle=\bar{u}_{N}\left(p^{\prime}, s^{\prime}\right)\left[A_{20}\left(Q^{2}\right) \gamma^{\left\{\mu P^{\nu}\right\}}+B_{20}\left(Q^{2}\right) \frac{i \sigma^{\{\mu \alpha} q_{\alpha} P^{\nu\}}}{2 m}+C_{20}\left(Q^{2}\right) \frac{q^{\{\mu} q^{\nu\}}}{m}\right] u_{N}(p, s)$,

where $\mathcal{O}_{\mathrm{V}}^{\mu \nu}=\bar{\psi} \gamma^{\left\{\mu_{i} \stackrel{\leftrightarrow}{D}\right.}{ }^{\nu\}} \psi$ and $A_{20}\left(Q^{2}\right), B_{20}\left(Q^{2}\right)$ and $C_{20}\left(Q^{2}\right)$ are the three GFFs. $Q^{2}=-q^{2}$ is the momentum transfer squared in Euclidean space. The second Mellin moment is given by $A_{20}(0)$, which gives the momentum fraction carried by a quark $\langle x\rangle_{q}$, while the total spin carried by a quark is given by $J_{q}=\frac{1}{2}\left[A_{20}(0)+B_{20}(0)\right]$. In Fig. 8 we compare lattice QCD results for the isovector $\langle x\rangle_{u-d}$ with results from phenomenological analyses. As can be seen, although individual results are precise, the spread among them is comparable to the accuracy achieved within lattice QCD. In the same figure we show results for the helicity and transversity moments. For the helicity lattice QCD results are in agreement with phenomenological determinations while for the transversity moment lattice QCD provide a valuable prediction.
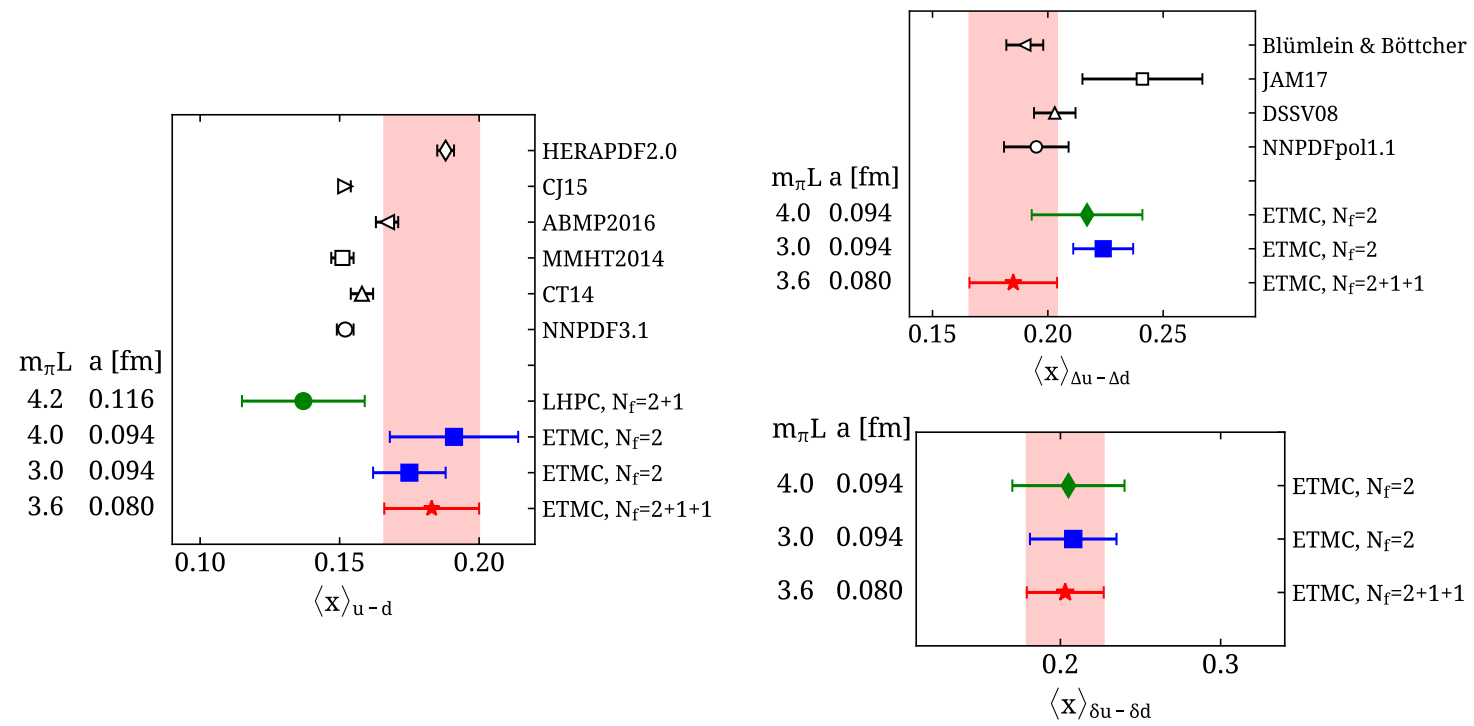

Figure 8: A comparison of recent lattice QCD and phenomenological results on the momentum fraction $\langle x\rangle_{u-d}$ (left), on the helicity moment $\langle x\rangle_{\Delta u-\Delta d}$ (upper right) and the transversity $\langle x\rangle_{\delta u-\delta d}$ (lower right). The error band is the one associate with the analysis of the twisted mass ensemble cB211.072.64.

While for isovector quantities only the connected part of the three-point contributes, for the isoscalar and thus for the determination of the individual flavor Mellin moments, we need to evaluate the disconnected part depicted in Fig. 5. The disconnected part is technically more difficult to compute and requiring large computational resources. It is only recently that we are able to compute these diagrams, which are needed for studying sea quarks effects. This has become possible using a combination of stochastic techniques, dilution and deflation of 
lower modes [13]. The intrinsic spin $\frac{1}{2} \Delta \Sigma^{q}$ carried by a quark of flavor $q$ is given by

$$
\Delta \Sigma_{q}\left(\mu^{2}\right)=\int_{0}^{1} d x\left[\Delta q\left(x, \mu^{2}\right)+\Delta \bar{q}\left(x, \mu^{2}\right)\right]=g_{A}^{q} .
$$

In Fig. 9 we show lattice QCD results on the intrinsic quark spin for the light and strange quarks. The former have contributions form both connected and disconnected parts, while the strange is purely disconnected. As can be seen, the disconnected contributions, although small, are non-zero and need to be included to reach agreement with the experimental values. Beyond

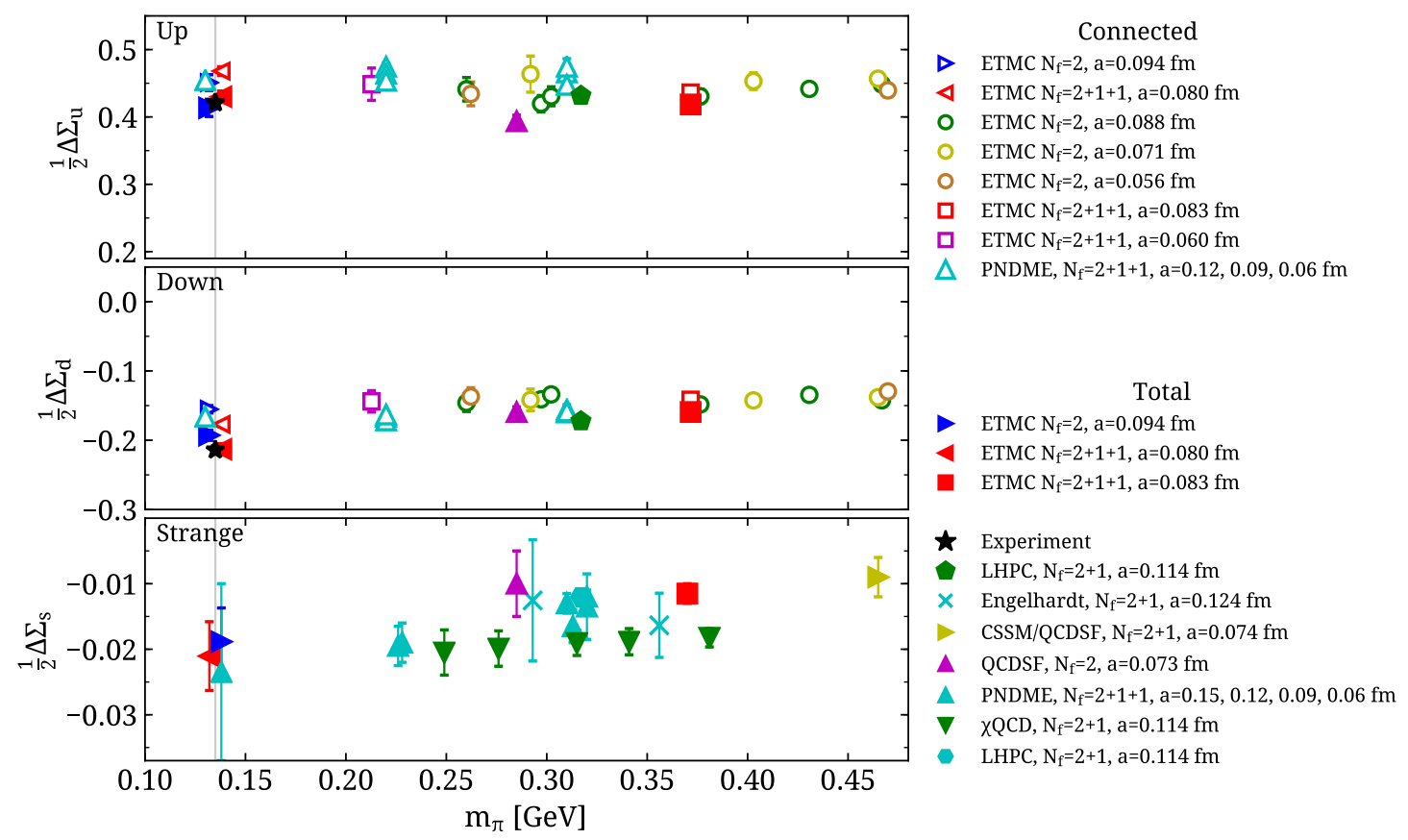

Figure 9: We show the intrinsic quark spin $\frac{1}{2} \Delta \Sigma^{q}$ for the $u$ (upper), the $d$ (center) and $s$ quarks (lower) as a function of the pion mass. Open symbols do not include the disconnected contribution.

the single favor axial and tensor charges one can extract the scalar charge and the closely related $\sigma$-terms defined as $\sigma_{q}=m_{q}\left\langle N\left|\bar{\psi}_{q} \psi_{q}\right| N\right\rangle$. The combination $\sigma_{\pi N}=1 / 2\left(\sigma^{u}+\sigma^{d}\right)$ constitutes one of the fundamental low-energy parameters paying a significant role for phenomenological studies of low energy scattering. The $\sigma$-terms are important for direct dark matter searches [14]. In Table 1 we summarize the nucleon charges for each quark flavor. Because of the phenomenological importance of $\sigma$-terms a number of groups have computed

\begin{tabular}{l|cccc}
\hline & $u$ & $d$ & $s$ & $c$ \\
\hline$g_{A}$ & $0.858(17)$ & $-0.428(17)$ & $-0.0450(71)$ & $-0.0098(34)$ \\
$g_{S}$ & $6.02(55)$ & $4.67(44)$ & $0.395(54)$ & $0.075(17)$ \\
$g_{T}$ & $0.716(28)$ & $-0.210(11)$ & $-0.00270(58)$ & $-0.00023(16)$ \\
$\sigma[\mathrm{MeV}]$ & $41.6(3.8)^{\dagger}$ & $39.8(5.5)$ & $107(22)$ \\
\hline
\end{tabular}

Table 1: Single flavor charges and $\sigma$-terms from the cB211.072.64 ensemble. ( $\dagger$ ) In the case of $u$ and $d$ quarks we give $\sigma_{\pi N}$ of relevance to phenomenology.

them. In Fig. 10 we compare lattice QCD and phenomenological results on $\sigma_{\pi N}$ and $\sigma_{s}$. The smaller value predicted by lattice QCD is in agreement with the original analysis that yielded 
$\sigma_{\pi N} \sim 45 \mathrm{MeV}$ [15] but it is in tension with recent analyses that yield larger values, such as an analysis based on the Roy-Steiner equations and experimental data on pionic atoms yielding a value of 59.1(3.5) $\mathrm{MeV}$ [16] that was confirmed using a large-scale fit of pionic-atom level shift and width data across the periodic table [17] as well as $\pi N$ scattering lengths from the low-energy data base [18]. Given the significant progress in the determination of $\sigma_{\pi N}$ both using experimental data [19-21] and lattice QCD this persisting tension needs to be further examined. Computing the $\pi N$ scattering lengths within lattice QCD will provide a crucial cross-check. For the charm $\sigma_{c}$ lattice QCD provides a valuable prediction.
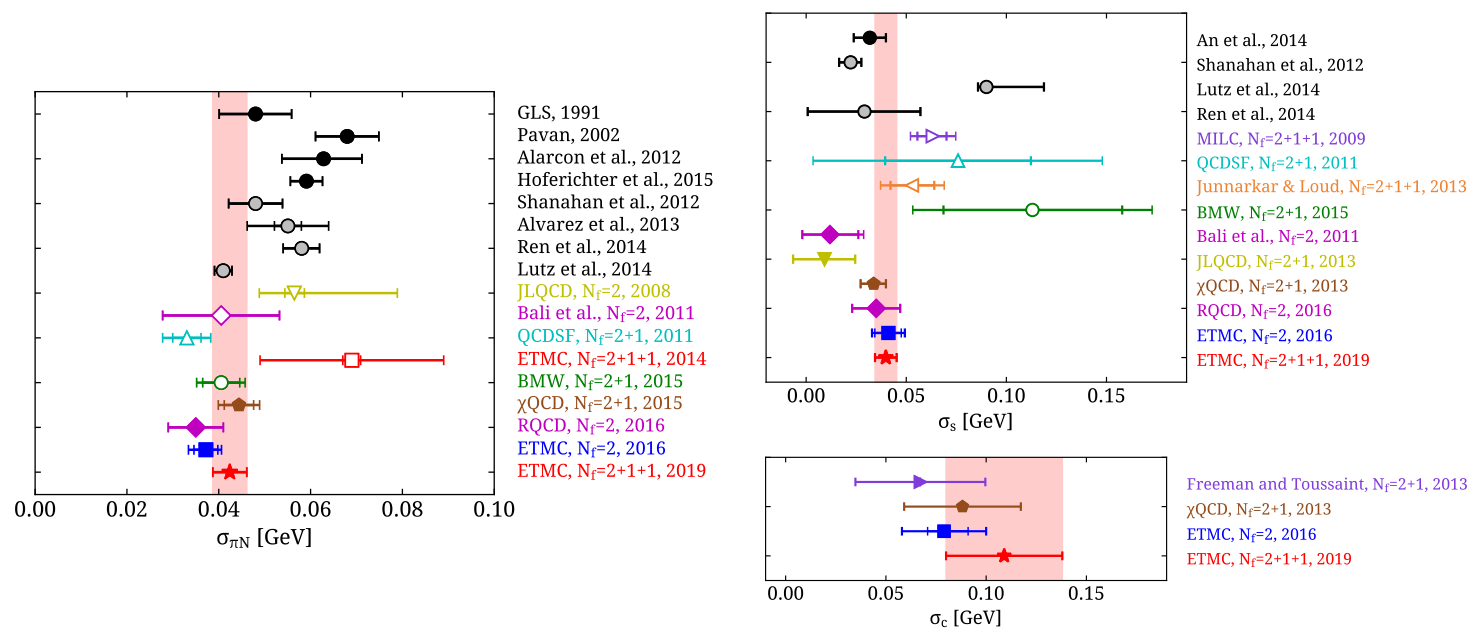

Figure 10: A comparison of recent lattice QCD and phenomenological results on the nucleon $\sigma_{\pi N}$-term fraction (left), on the strange $\sigma_{c}$-term (upper right) and the charm $\sigma_{c}$-term (lower right). The error band is the one associate with the analysis of the twisted mass ensemble cB211.072.64. Grey circles are phenomenological studies using as input lattice data.

\subsection{Electromagnetic form factors}

The nucleon electromagnetic form factors are fundamental quantities characterizing the structure of the nucleon that have been extensively studied both theoretically and experimentally. A recent major experimental development is the measurement of the proton charge radius with electron scattering experiments at Jefferson lab [22] that confirms the smaller value measured by the Lamb shift in muonic hydrogen at PSI [23], the point to the resolution of the so-called proton radius puzzle. Computing such fundamental properties as the proton radius directly from QCD constitutes a milestone of nuclear physics and of lattice QCD.

The nucleon matrix element of the electromagnetic current is parameterized in terms of the Dirac $\left(F_{1}\right)$ and Pauli $\left(F_{2}\right)$ form factors as

$$
\left\langle N\left(p^{\prime}, s^{\prime}\right)\left|j_{\mu}\right| N(p, s)\right\rangle=\sqrt{\frac{m_{N}^{2}}{E_{N}\left(\vec{p}^{\prime}\right) E_{N}(\vec{p})}} \bar{u}_{N}\left(p^{\prime}, s^{\prime}\right)\left[\gamma_{\mu} F_{1}\left(q^{2}\right)+\frac{i \sigma_{\mu \nu} q^{\nu}}{2 m_{N}} F_{2}\left(q^{2}\right)\right] u_{N}(p, s) .
$$

$N(p, s)$ is the nucleon state with initial (final) momentum $p\left(p^{\prime}\right)$ and spin $s\left(s^{\prime}\right), u_{N}$ is the nucleon spinor, $E_{N}(\vec{p})\left(E_{N}\left(\vec{p}^{\prime}\right)\right)$ the initial (final) energy and $m_{N}$ the nucleon mass. The electric and magnetic Sachs form factors $G_{E}\left(q^{2}\right)$ and $G_{M}\left(q^{2}\right)$ are alternative Lorentz invariant quantities that can be expressed in terms of $F_{1}\left(q^{2}\right)$ and $F_{2}\left(q^{2}\right)$ via the relations,

$$
G_{E}\left(q^{2}\right)=F_{1}\left(q^{2}\right)+\frac{q^{2}}{4 m_{N}^{2}} F_{2}\left(q^{2}\right), \quad G_{M}\left(q^{2}\right)=F_{1}\left(q^{2}\right)+F_{2}\left(q^{2}\right)
$$


In Fig. 11 we show results on the electric and magnetic form factors for the neutron [13] using the twisted mass ensemble cB211.072.64. The disconnected contributions are included. As can be seen, lattice QCD provides more precise results on the neutron electric form factor than experiment, while for the magnetic we observe a nice agreement for $Q^{2}>0.2 \mathrm{GeV}^{2}$. Why there are small discrepancies at smaller $Q^{2}$ is under investigation.
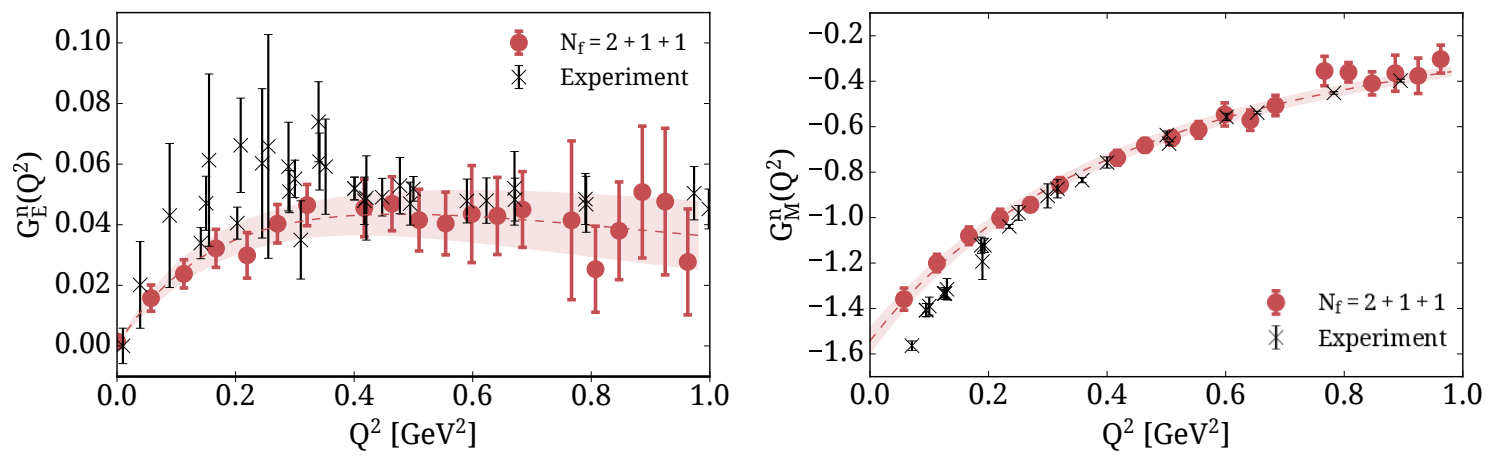

Figure 11: Neutron electric (left) and magnetic (right) form factors as a function of $Q^{2}$. Filled circles show the lattice QCD results obtained using the cB211.072.64 ensemble [13] and black crosses are experimental results (see Ref. [13] for the the detailed bibliography on the experimental results). The fits to lattice QCD results use the Galster-like form for the electric form factor and a dipole form for the magnetic.

The role of the strange quarks in the proton can be probed through the strange electromagnetic form factors. Parity violating electron-proton elastic scattering events probing the interference of photons and Z-bosons exchanges enable the measurement of the strange form factors and weak charge of the proton. An accurate determination of the neutral-weak vector form factor in combination with the electromagnetic form factors are needed in order to put constraints on new physics beyond the standard model (SM). In Fig. 12 we show results on the strange electromagnetic form factors [24]. As can be seen, the strange magnetic form factor is negative yielding a magnetic moment of -0.01 to -0.02 . This provides the most accurate determination of these quantities.
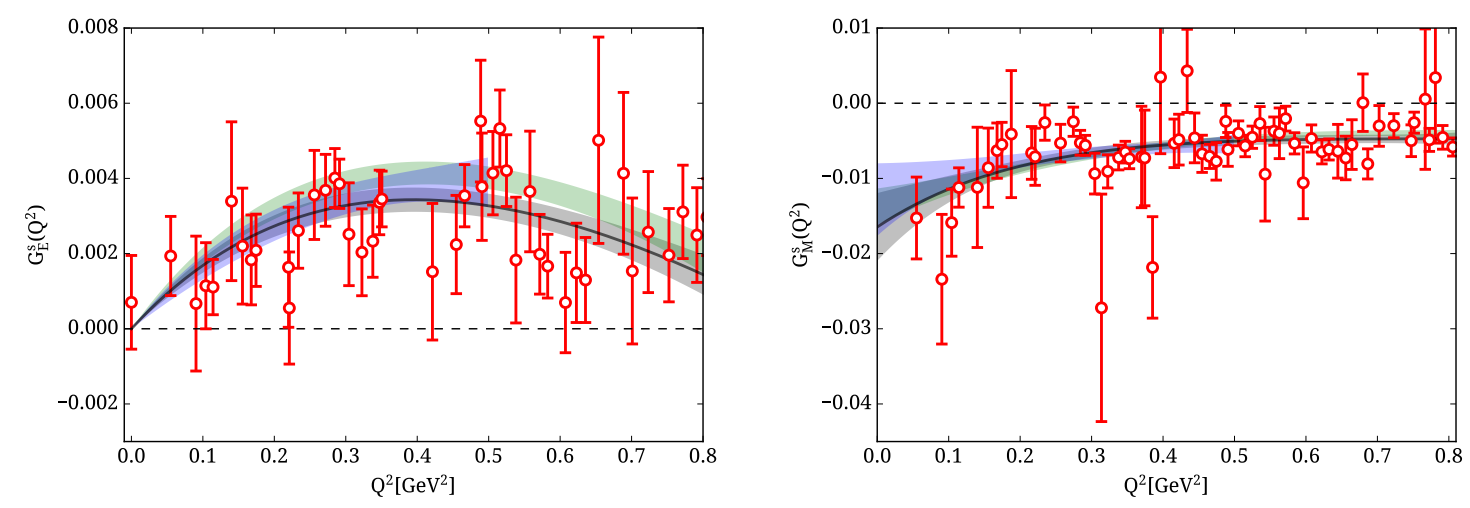

Figure 12: Strange electric (left) and magnetic (right) form factors as a function of $Q^{2}$. The different coloured band show systematics due to using different fit range and the plateau versus the summation determination [24]. 


\subsection{Direct evaluation of PDFs}

A calculation of PDFs from first principles is a valuable addition to the global fitting analyses and of crucial importance for the deeper understanding of the inner structure of hadrons. The non-perturbative nature of PDFs makes lattice QCD an ideal ab initio formulation to determine them. A novel method to extract parton distribution functions from lattice QCD was proposed recently by $\mathrm{Ji}[2]$. It is based on considering matrix elements probing purely spatial correlations, making them accessible within the Euclidean formulation of lattice QCD. It is based on the Large Momentum Effective Theory (LaMET) that enables the matching of lattice results to the infinity momentum frame (IFM). Quasi-PDFs and IFM PDFs have the same infrared physics and thus the matching can be done in perturbation theory i.e. UV regularization is necessarily taken first, before the infinite momentum limit. Quasi-PDFs are defined as

$$
\tilde{q}\left(x, P_{3}\right)=\int_{-z_{\max }}^{+z_{\max }} \frac{d z}{4 \pi} e^{-i x P_{3} z}\langle N|\bar{\psi}(0, z) \mathcal{G} W(z, 0) \psi(0,0)| N\rangle,
$$

where $|N\rangle$ represents a nucleon, which is boosted in the z-direction with momentum $P=\left(P_{0}, 0,0, P_{3}\right)$. On the lattice, quasi-PDFs are thus computed using matrix elements of non-local operators containing a straight Wilson line of finite length $z$, that varies from 0 to some maximum value, $z_{\max } \cdot$

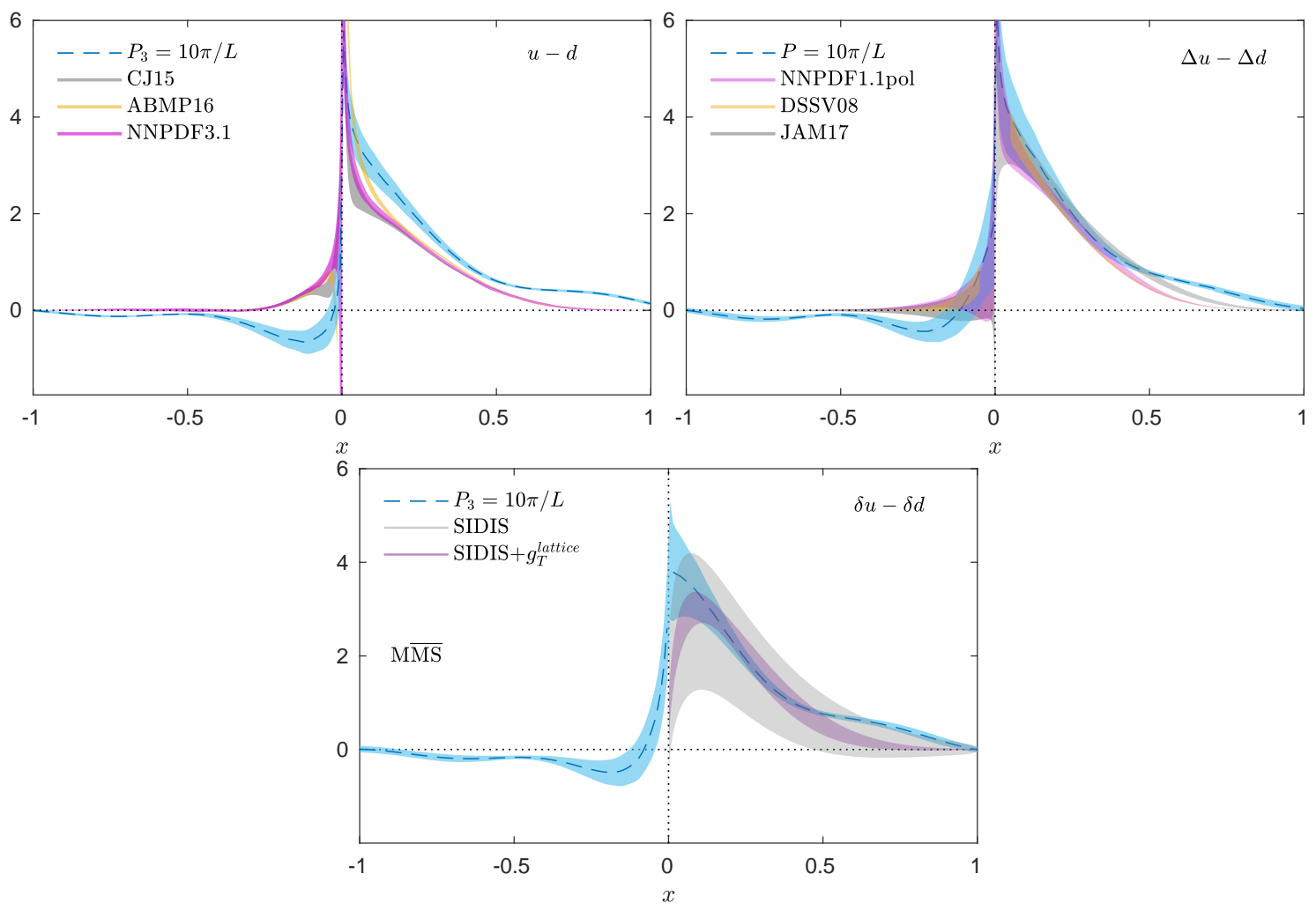

Figure 13: Unpolarized PDF (upper left), helicity PDF (upper right) and transversity PDF (lower) (blue curve). The global fits of Refs. [29-31] (unpolarized) , Refs. [3234] (helicity), Refs. [35] (transversity) are shown for qualitative comparison.

Quasi-PDFs are matched to the physical PDFs through the factorization

$$
q(x, \mu)=\int_{-\infty}^{\infty} \frac{d \xi}{|\xi|} C\left(\xi, \frac{\mu}{P_{3}}\right) \tilde{q}\left(\frac{x}{\xi}, \mu, P_{3}\right)+O\left(\frac{m_{N}^{2}}{P_{3}^{2}}, \frac{\Lambda_{Q C D}^{2}}{P_{3}^{2}}\right),
$$


where $q(x, \mu)$ is the light-cone PDF at an energy scale $\mu$ and $C$ is the matching kernel, which can be computed perturbatively and has so far been evaluated to one-loop level.

A first attempt to compute directly PDFs was done by LP3 [25] and ETMC [26]. In Fig. 13 we show the most recent results computed by ETMC using an ensemble simulated at the physical pion mass $[27,28]$. The errors shown are statistical only, and significant effort is needed to properly quantify systematic uncertainties present in the various steps of the analysis. Nevertheless, already lattice QCD results can describe the general features and provide a more accurate determination of the transversity PDF.

\section{Conclusion}

Precision nucleon structure from lattice QCD is now possible due to two major developments: i) simulations using dynamical light, strange and charm quarks with their masses fixed to the physical value are available thanks to algorithmic advances and larger computers, and ii) computation of both connected and disconnected contributions to sufficient accuracy is feasible due to advanced techniques and access to GPUs. This progress will continue with the advent of exascale computers expected in the next couple of years.

A number of collaborations are computing key quantities such as the first and second Mellin moments reproducing the nucleon axial charge and providing a prediction for the tensor charge and second transversity moment. This enables cross-checks among the different formulations. We expect more precision results to emerge as systematic errors are investigated and taken into account. We also expect that more demanding quantities to become amenable to a lattice QCD computation. A recent example is the direct computation of parton distribution functions within lattice QCD. The results produced already are very promising and a number of complementary approaches are being advanced with good prospects for improvements.

\section{Acknowledgements}

I would like to thank all members of the Extended Twisted Mass Collaboration (ETMC) for a very constructive and enjoyable collaboration. In particular, I would like to thank my close collaborators S. Bacchio, K. Cichy, M. Constantinou, J. Finkenrath, K. Hadjiyiannakou, K. Jansen, G. Koutsou, A. Scapellato, F. Steffens and A. Vaquero Aviles-Casco, without whom this work would not be possible.

Funding information We acknowledge funding from the Horizon 2020 research and innovation program of the European Union under the Marie Sklodowska-Curie grant agreement No 642069 and from the COMPLEMENTARY/0916/0015 project funded by the Cyprus Research Promotion Foundation. Results were obtained using Piz Daint at Centro Svizzero di Calcolo Scientifico (CSCS), via the project with id s702. We thank the staff of CSCS for access to the computational resources and for their constant support. The Gauss Centre for Supercomputing e.V. (www.gauss-centre.eu) funded the project pr74yo by providing computing time on the GCS Supercomputer SuperMUC at Leibniz Supercomputing Centre (www.lrz.de). In addition, this work used computational resources from Extreme Science and Engineering Discovery Environment (XSEDE), which is supported by National Science Foundation grant number TGPHY170022. 


\section{References}

[1] A. Accardi et al., Electron Ion Collider: The Next QCD Frontier, Eur. Phys. J. A52(9), 268 (2016), doi:10.1140/epja/i2016-16268-9, 1212.1701.

[2] X. Ji, Parton Physics on a Euclidean Lattice, Phys. Rev. Lett. 110, 262002 (2013), doi:10.1103/PhysRevLett.110.262002, 1305.1539.

[3] B. Joó, C. Jung, N. H. Christ, W. Detmold, R. Edwards, M. Savage and P. Shanahan, Status and Future Perspectives for Lattice Gauge Theory Calculations to the Exascale and Beyond, Eur. Phys. J. A55(11), 199 (2019), doi:10.1140/epja/i2019-12919-7, 1904.09725.

[4] C. Alexandrou, S. Bacchio, J. Finkenrath, A. Frommer, K. Kahl and M. Rottmann, Adaptive Aggregation-based Domain Decomposition Multigrid for Twisted Mass Fermions, Phys. Rev. D94(11), 114509 (2016), doi:10.1103/PhysRevD.94.114509, 1610.02370.

[5] C. Alexandrou, S. Bacchio and J. Finkenrath, Multigrid approach in shifted linear systems for the non-degenerated twisted mass operator, Comput. Phys. Commun. 236, 51 (2019), doi:10.1016/j.cpc.2018.10.013, 1805.09584.

[6] S. Dürr, Validity of ChPT - is $M_{\pi}=135 \mathrm{MeV}$ small enough ?, PoS LATTICE2014, 006 (2015), doi:10.22323/1.214.0006, 1412.6434.

[7] G. Colangelo, A. Fuhrer and S. Lanz, Finite volume effects for nucleon and heavy meson masses, Phys. Rev. D82, 034506 (2010), doi:10.1103/PhysRevD.82.034506, 1005.1485.

[8] R. Aaij et al., Observation of the doubly charmed baryon $\Xi_{c c}^{++}$, Phys. Rev. Lett. 119(11), 112001 (2017), doi:10.1103/PhysRevLett.119.112001, 1707.01621.

[9] A. Ocherashvili et al., Confirmation of the double charm baryon Xi+(cc)(3520) via its decay to $p D+K$-, Phys. Lett. B628, 18 (2005), doi:10.1016/j.physletb.2005.09.043, hep-ex/0406033.

[10] C. Alexandrou and C. Kallidonis, Low-lying baryon masses using $N_{f}=2$ twisted mass clover-improved fermions directly at the physical pion mass, Phys. Rev. D96(3), 034511 (2017), doi:10.1103/PhysRevD.96.034511, 1704.02647.

[11] C. Alexandrou, S. Bacchio, M. Constantinou, J. Finkenrath, K. Hadjiyiannakou, K. Jansen, G. Koutsou and A. Vaquero Aviles-Casco, The nucleon axial, tensor and scalar charges and $\sigma$-terms in lattice QCD (2019), 1909.00485.

[12] M. Radici and A. Bacchetta, First Extraction of Transversity from a Global Analysis of Electron-Proton and Proton-Proton Data, Phys. Rev. Lett. 120(19), 192001 (2018), doi:10.1103/PhysRevLett.120.192001, 1802.05212.

[13] C. Alexandrou, S. Bacchio, M. Constantinou, J. Finkenrath, K. Hadjiyiannakou, K. Jansen, G. Koutsou and A. Vaquero Aviles-Casco, Proton and neutron electromagnetic form factors from lattice QCD, Phys. Rev. D100(1), 014509 (2019), doi:10.1103/PhysRevD.100.014509, 1812.10311.

[14] J. Giedt, A. W. Thomas and R. D. Young, Dark matter, the CMSSM and lattice QCD, Phys. Rev. Lett. 103, 201802 (2009), doi:10.1103/PhysRevLett.103.201802, 0907.4177.

[15] J. Gasser, H. Leutwyler and M. E. Sainio, Sigma term update, Phys. Lett. B253, 252 (1991), doi:10.1016/0370-2693(91)91393-A. 
[16] M. Hoferichter, J. Ruiz de Elvira, B. Kubis and U.-G. Meißner, High-Precision Determination of the Pion-Nucleon $\sigma$ Term from Roy-Steiner Equations, Phys. Rev. Lett. 115, 092301 (2015), doi:10.1103/PhysRevLett.115.092301, 1506.04142.

[17] E. Friedman and A. Gal, The pion-nucleon $\sigma$ term from pionic atoms, Phys. Lett. B792, 340 (2019), doi:10.1016/j.physletb.2019.03.036, 1901.03130.

[18] J. Ruiz de Elvira, M. Hoferichter, B. Kubis and U.-G. Meißner, Extracting the $\sigma$-term from low-energy pion-nucleon scattering, J. Phys. G45(2), 024001 (2018), doi:10.1088/13616471/aa9422, 1706.01465.

[19] M. Hoferichter, J. Ruiz de Elvira, B. Kubis and U.-G. Meißner, Roy-Steinerequation analysis of pion-nucleon scattering, Phys. Rept. 625, 1 (2016), doi:10.1016/j.physrep.2016.02.002, 1510.06039.

[20] M. Hoferichter, C. Ditsche, B. Kubis and U. G. Meissner, Dispersive analysis of the scalar form factor of the nucleon, JHEP 06, 063 (2012), doi:10.1007/JHEP06(2012)063, 1204. 6251.

[21] J. M. Alarcon, J. Martin Camalich and J. A. Oller, The chiral representation of the $\pi N$ scattering amplitude and the pion-nucleon sigma term, Phys. Rev. D85, 051503 (2012), doi:10.1103/PhysRevD.85.051503, 1110.3797.

[22] W. Xiong et al., A small proton charge radius from an electron-proton scattering experiment, Nature 575(7781), 147 (2019), doi:10.1038/s41586-019-1721-2.

[23] R. Pohl et al., The size of the proton, Nature 466, 213 (2010), doi:10.1038/nature09250.

[24] C. Alexandrou, S. Bacchio, M. Constantinou, J. Finkenrath, K. Hadjiyiannakou, K. Jansen and G. Koutsou, Nucleon strange electromagnetic form factors (2019), 1909.10744.

[25] H.-W. Lin, J.-W. Chen, S. D. Cohen and X. Ji, Flavor Structure of the Nucleon Sea from Lattice QCD, Phys. Rev. D91, 054510 (2015), doi:10.1103/PhysRevD.91.054510, 1402. 1462.

[26] C. Alexandrou, K. Cichy, V. Drach, E. Garcia-Ramos, K. Hadjiyiannakou, K. Jansen, F. Steffens and C. Wiese, Lattice calculation of parton distributions, Phys. Rev. D92, 014502 (2015), doi:10.1103/PhysRevD.92.014502, 1504.07455.

[27] C. Alexandrou, K. Cichy, M. Constantinou, K. Jansen, A. Scapellato and F. Steffens, Transversity parton distribution functions from lattice QCD, Phys. Rev. D98(9), 091503 (2018), doi:10.1103/PhysRevD.98.091503, 1807.00232.

[28] C. Alexandrou, K. Cichy, M. Constantinou, K. Jansen, A. Scapellato and F. Steffens, LightCone Parton Distribution Functions from Lattice QCD, Phys. Rev. Lett. 121(11), 112001 (2018), doi:10.1103/PhysRevLett.121.112001, 1803.02685.

[29] S. Alekhin, J. Blümlein, S. Moch and R. Placakyte, Parton distribution functions, $\alpha_{s}$, and heavy-quark masses for LHC Run II, Phys. Rev. D96(1), 014011 (2017), doi:10.1103/PhysRevD.96.014011, 1701.05838.

[30] R. D. Ball et al., Parton distributions from high-precision collider data, Eur. Phys. J. C77(10), 663 (2017), doi:10.1140/epjc/s10052-017-5199-5, 1706.00428.

[31] A. Accardi, L. T. Brady, W. Melnitchouk, J. F. Owens and N. Sato, Constraints on large-x parton distributions from new weak boson production and deep-inelastic scattering data, Phys. Rev. D93(11), 114017 (2016), doi:10.1103/PhysRevD.93.114017, 1602.03154. 
[32] D. de Florian, R. Sassot, M. Stratmann and W. Vogelsang, Extraction of SpinDependent Parton Densities and Their Uncertainties, Phys. Rev. D80, 034030 (2009), doi:10.1103/PhysRevD.80.034030, 0904.3821.

[33] E. R. Nocera, R. D. Ball, S. Forte, G. Ridolfi and J. Rojo, A first unbiased global determination of polarized PDFs and their uncertainties, Nucl. Phys. B887, 276 (2014), doi:10.1016/j.nuclphysb.2014.08.008, 1406.5539.

[34] J. J. Ethier, N. Sato and W. Melnitchouk, First simultaneous extraction of spin-dependent parton distributions and fragmentation functions from a global QCD analysis, Phys. Rev. Lett. 119(13), 132001 (2017), doi:10.1103/PhysRevLett.119.132001, 1705.05889.

[35] H.-W. Lin, W. Melnitchouk, A. Prokudin, N. Sato and H. Shows, First Monte Carlo Global Analysis of Nucleon Transversity with Lattice QCD Constraints, Phys. Rev. Lett. 120(15), 152502 (2018), doi:10.1103/PhysRevLett.120.152502, 1710.09858. 